\title{
Evidence Based Development of a Novel Lateral Fibula Plate (VariAx Fibula) Using a Real CT Bone Data Based Optimization Process During Device Development
}

\author{
Arndt P. Schulz ${ }^{*}, 1$, Nils Reimers ${ }^{2}$, Felix Wipf ${ }^{2}$, Michel Vallotton ${ }^{2}$, Serena Bonaretti ${ }^{3}$, Nina Kozic ${ }^{3}$, \\ Mauricio Reyes ${ }^{3}$ and Benjamin J Kienast ${ }^{4}$ \\ ${ }^{1}$ University Hospital Lübeck, Germany \\ ${ }^{2}$ StrykerOsteosynthesis, Schönkirchen, Germany \\ ${ }^{3}$ Institute for Surgical Technology and Biomechanics, Bern, Switzerland \\ ${ }^{4}$ Trauma Center Hamburg, Germany
}

\begin{abstract}
Development of novel implants in orthopaedic trauma surgery is based on limited datasets of cadaver trials or artificial bone models. A method has been developed whereby implants can be constructed in an evidence based method founded on a large anatomic database consisting of more than 2.000 datasets of bones extracted from CT scans. The aim of this study was the development and clinical application of an anatomically pre-contoured plate for the treatment of distal fibular fractures based on the anatomical database.

48 Caucasian and Asian bone models (left and right) from the database were used for the preliminary optimization process and validation of the fibula plate. The implant was constructed to fit bilaterally in a lateral position of the fibula. Then a biomechanical comparison of the designed implant to the current gold standard in the treatment of distal fibular fractures (locking $1 / 3$ tubular plate) was conducted. Finally, a clinical surveillance study to evaluate the grade of implant fit achieved was performed. The results showed that with a virtual anatomic database it was possible to design a fibula plate with an optimized fit for a large proportion of the population. Biomechanical testing showed the novel fibula plate to be superior to $1 / 3$ tubular plates in 4-point bending tests. The clinical application showed a very high degree of primary implant fit. Only in a small minority of cases further intra-operative implant bending was necessary. Therefore, the goal to develop an implant for the treatment of distal fibular fractures based on the evidence of a large anatomical database could be attained. Biomechanical testing showed good results regarding the stability and the clinical application confirmed the high grade of anatomical fit.
\end{abstract}

Keywords: Evidence based implant design, fibula fracture, locked plate, bone database, distal, biomechanical, anatomical.

\section{INTRODUCTION}

In current trauma and orthopaedic research bone shape variability within a specific population has been rarely investigated and used to optimize implant design. Implants are commonly designed by evaluating implant bone fitting on a limited dataset of cadaver bones or even artificial bone models $[1,2]$. On the other hand, more and more precontoured implants are in use in orthopaedic trauma surgery [3-8]. These are designed for a specified anatomic location. Due to the pre-contoured shape with an anatomic fit, these plates can supposedly be applied in a minimally invasive fashion [9-13]. In a recent biomechanical study it has been proven that the bone shape fit of these "anatomically precontoured" plates can be as low as $13 \%$ [14]. During the developmental process of such implants, the diversity of the target population (e.g. age, gender, ethnical background etc.) is often not taken into account.

*Address correspondence to this author at the University Lübeck, Department of Musculoskeletal Surgery, Ratzeburger Allee 160, D-23538 Lübeck, Germany; Tel: +49-451-5004730;

E-mail: schulz@biomechatronics.de
However, extrapolating the findings gained by such tests to the whole target population may lead to implants that fit only some patients (for an example see Fig. 1). The importance of determining the shape of the implant that best fits a majority of the population is paramount, both from a clinical and an economical perspective [15].

The mismatch leads to well known clinical complications resulting from inadequate fixation [16]. Further intraoperative bending of those implants weakens the biomechanical stability.

The theoretical advantage of implants designed by using a three-dimensional bone model is accuracy, easy fitting assessment and implant shape optimization, comprising a variety of the target population. The biomechanical properties of such implants can be optimized during the design process by virtual methods.

A large anatomical virtual bone database for implant development and evaluation has been created [15] for this purpose. This has been initially used for the evaluation and improvement of implants used for the treatment of proximal tibial fractures [17]. The aim of this study was the design of 

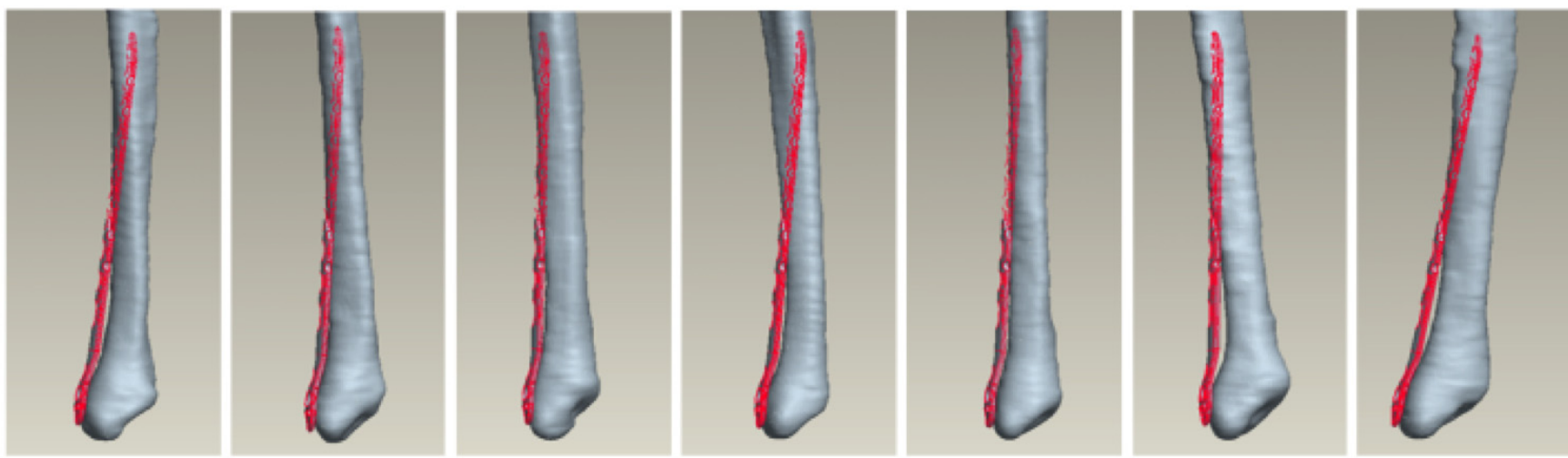

Fig. (1). Matching of the previous generation of fibular plates matched onto 7 randomly assigned fibulae from a virtual database without plate shape bending.

a distal fibula plate with a good primary fit in a majority of the target population. During this study specific design details, such as the need of dedicated left and right plates and the need of a plate twist in the shaft portion were investigated. Biomechanical testing in comparison to the current gold standard was performed. The clinical fitting of those newly designed plates was evaluated.

The results of the study are presented up to the clinical use in selected cases. The testeddistal fibula plate is now commercially available (VariAx Distal Lateral Fibula Locking Plate, Stryker, Schönkirchen, Germany).

\section{MATERIALS \& METHODS}

\section{Evidence Based Implant Design}

The initial dataset consisted of 48 fibula surface models extracted from computer tomography (CT) data comprising a variety of human population (20 Caucasian and 28 Asian). The CT scans were acquired worldwide during regular CT examinations for various medical conditions excluding bone and joint diseases. The process of data acquisition was fully anonymized and controlled by a governmental data protection agency (DatenschutzbeauftragterStadt Bremen, Bremen, Germany).

To compensate for different positioning during CT acquisition, segmented bones (Amira $\mathrm{C}$, Visage Imaging) were spatially aligned via rigid registration. Statistical shape modeling was then performed as explained in [15]. The constructed statistical model consisting of average bone and its modes of variation was then used to build a virtual bone database (Fig. 2).

The advanced implant fitting software developed in collaboration with the Institute for Surgical Technology and Biomechanics in Bern, Switzerland [17], was used to determine the best matching position between the bones and the implant. The automatic fitting method starts with an initial implant positioning for the fitting process, which was chosen manually and separately for each bone in order to ensure the best possible fitting. In addition, the initialization was verified automatically using a grid of dimensions $4 \times 4 \mathrm{~mm}^{2}$ and spacing $0.5 \mathrm{~mm}$ for different implant positioning. In order to find plausible implant fitting, additional fitting constraints were incorporated to the optimization of the fitting procedure. Collision constraint was used to ensure that no points of the implant mesh fell inside the bone model. Furthermore, in order to favor colinearity with the main bone axis, parallelism constraint was included. Finally, plateau constraint was used to ensure that no points of the implant mesh took place above the bone mesh plateau. The best fitting was then chosen as the one giving the minimal root-mean-square distance (computed for the whole implant surface).

Investigation of implant to bone surface fitting distance maps were enabled for optimized implant design. The shape of the VariAx Distal Lateral Fibula Plate was designed to anatomically best fit a majority of the target population. The principles of locked plating were used in the design of the plate screw system $[5,7,18]$. Finally, the implant shape was validated by the statistical shape model fitting (VBDB), to ensure anatomical and ethnical variety.

\section{Biomechanical Evaluation}

To evaluate the fatigue strength of the plate design the newly developed implant was tested against the current "gold standard" in this field, a titanium locking compression (LC) 1/3 Tubular Plate (Synthes, Switzerland). Bone surrogate materialwas chosen to simulate a comminuted fibula fracture by creating an $11 \mathrm{~mm}$ gap. The bone surrogate was manufactured in a simplified anatomical shape (Fig. 3). The LC 1/3 Tubular Plate was contoured to fit the bone model shape while the VariAx Fibula plate was used in its anatomically pre-shaped design and did not require additional contouring (Fig. 3, Table 1). Contouring of the LC $1 / 3$ Tubular Plate led to a convergent trajectory of the two distal screws. The four distal VariAx screws were inserted with the maximum diverging angulation of 15 degrees orthogonal to the plate surface.

Aservo-hydraulic testing machine was used to applya cyclic uniformly bending moment to the over the entire plate length until failure occurred (Fig. 4).

In the first test series, fatigue strength was tested. The aim of a fatigue test is to determine the plate strength. As hardware failure was intended to test bone surrogate with a high strength was chosen to generate plate failure.

The clinical significance of this test is: The higher the fatigue strength the higher the safety against implant failure due to delayed fracture healing. 


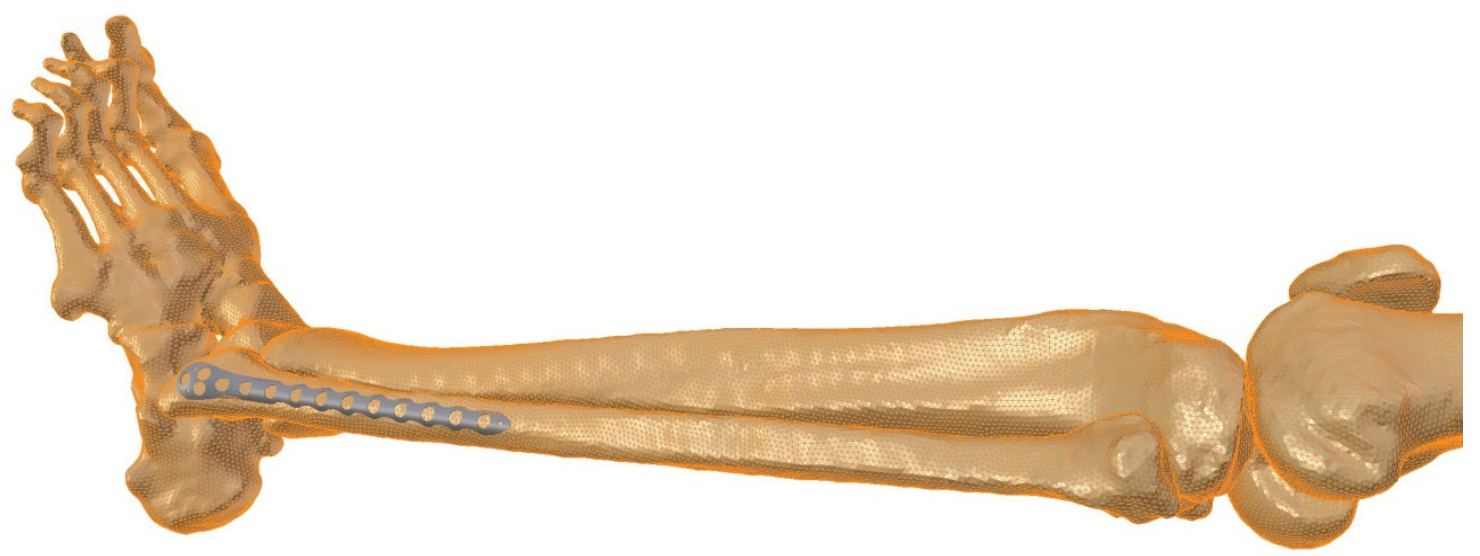

Fig. (2). The constructed statistical model consisting of average bone, for demonstration here superimposed a fibula plate.

To determine the fatigue strength of the plate up to half a million load cycles were applied. This corresponds to approximately six months of in vivo loading time [19]. With at least six samples per test group, the fatigue strength was calculated following the statistical procedure described in ASTM STP 731 [20].

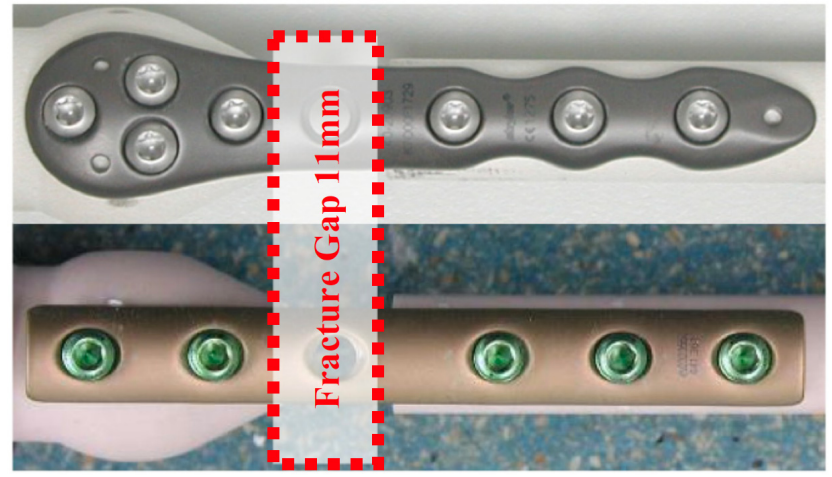

Fig. (3). Implants chosen in the biomechanical testing, top: novel VariAx Plate, bottom: 1/3 Tubular LC Plate.

Table 1. Parameters of the Used Implants

\begin{tabular}{|c|c|c|}
\hline Implant & $\begin{array}{c}\text { Locking } \\
\text { Screw }\end{array}$ & $\begin{array}{c}\text { Material } \\
\text { Anodization }\end{array}$ \\
\hline \hline $\begin{array}{c}\text { VariAx Distal Lateral Fibula Plate } \\
\text { (Stryker) }\end{array}$ & $\begin{array}{c}3.5 \times 16 \mathrm{~mm}(\mathrm{n}=7) \\
\text { Polyaxial }\end{array}$ & $\begin{array}{c}\text { Titanium } \\
\text { Anodization Type II }\end{array}$ \\
\hline $\begin{array}{c}\text { LC1/3 Tubular Plate } \\
\text { (Synthes) }\end{array}$ & $\begin{array}{c}3.5 \times 20 \mathrm{~mm}(\mathrm{n}=5) \\
\text { Monoaxial }\end{array}$ & $\begin{array}{c}\text { Titanium } \\
\text { Anodization Type III }\end{array}$ \\
\hline
\end{tabular}

In a further test series cut-out strength was determined. The cut-out test focuses on the interface between the screws and the bone. To simulate the worst case interface a poor quality bone model was chosen for the distal fragment.

The clinical significance of this test is: The higher the cut-out strength of the screw-plate construct the lower the risk for in vivo failure of the screw/bone interface.

Bone simulation material made of polyurethane foam with a density of $80 \mathrm{~kg} / \mathrm{m}^{3}$ was used. A cyclic load was applied in a staircase procedure starting at $10 \mathrm{~N}$. If no failure occurred the load was increased by $10 \mathrm{~N}$ after every 1.000 load cycles. Failure wasdefined as a misalignment of at least 10 degrees. Three samples of each test group were tested.

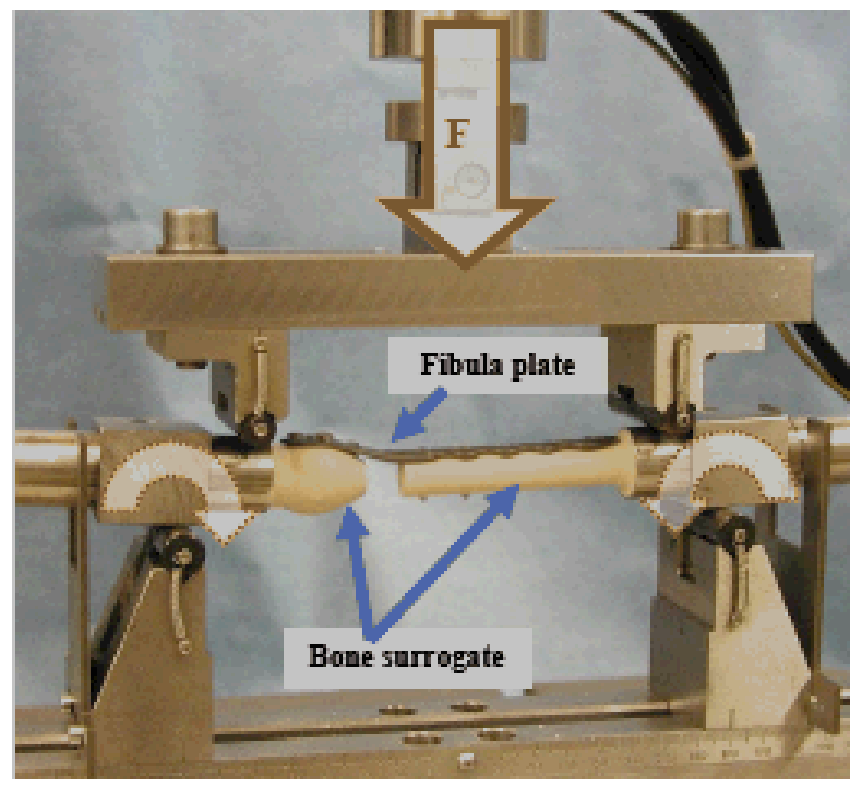

Fig. (4). Biomechanical test setup of the four point bending tests, $F$ $=$ applied cyclic load that results into a uniformly bending moment over the entire plate length.

\section{Clinical Investigation of the New VariAx Distal Fibula Plate}

After FDA and CE approval of the new VariAx distal fibula plate the surgical trays where delivered by Stryker to 21 centres (26 surgeons) in 5 countries (Fig. 5). Only data that was not patient related was stored in a database. The dataset included the fracture type, exact details of the implants used and details about the procedure, including necessary bending of the plate and the use of additional lag screws, and the overall satisfaction of the surgeon with the novel device. The statistical evaluation was performed with SPSS software (Statistical Package for Social Sciences, version 16, SPSS Inc., Chicago, USA).

The study period was 8 weeks, with 57 cases included in the study.

There was a wide variation in the age range. AO type B fractures were found in $47 \%$ of cases (Figs. 6, 7). 


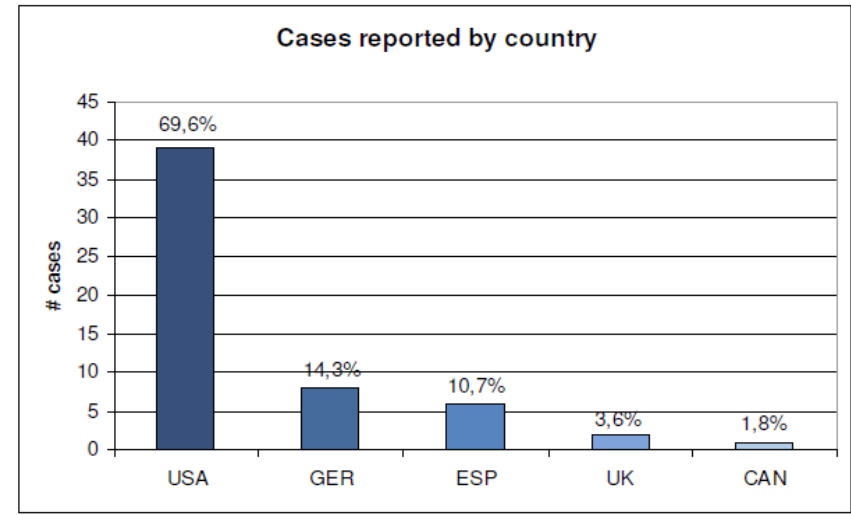

Fig. (5). Participating countries and sample sizes.

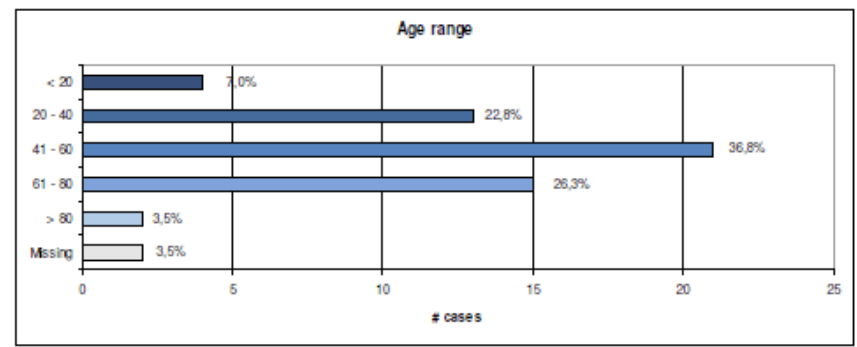

Fig. (6). Age range distribution $(n=57)$.

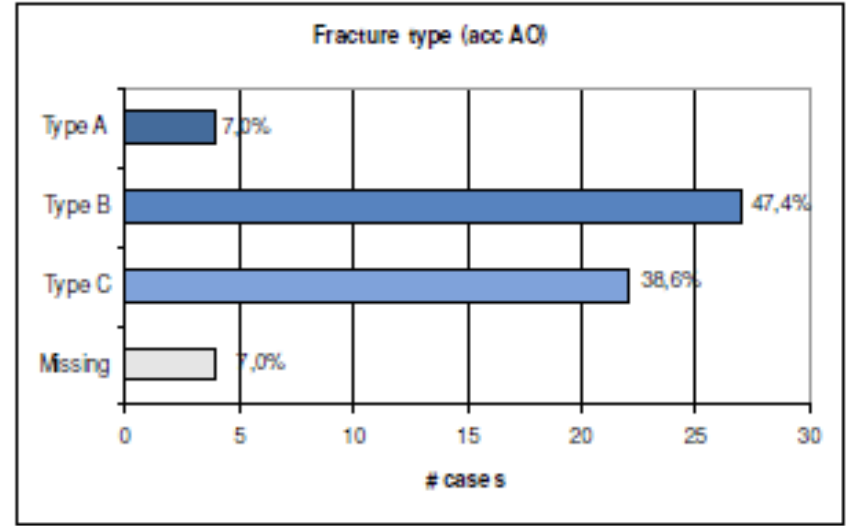

Fig. (7). Fracture type as classified by the AO system $(n=53$; in 4 cases no data was available).

\section{RESULTS}

\section{Virtual Implant Design}

With the aid of the virtual bone database it was possible to design a plate with a maximum fit on a statistical bone shape for the target population. The anatomical evaluation showed that fitting results cannot be improved by using a left and right version and that there is no twist needed with the appropriate contour (Fig. 8). Furthermore, it became clear that it would be possible to fit the design with two parallel holes in the metaphyseal area due to the broad shape in the distal area (Fig. 9). That type of shape made it possible to reduce the implant thickness in the metaphyseal area from 2.0 to $1.3 \mathrm{~mm}$.

The quality of the implant fitting was evaluated on each of the bones generated with the statistical model.

For each bone the software output consisted of:
- $\quad$ List of distances implant points to bone surface (mm)

- $\quad$ Fitted data in STL format

- $\quad \mathrm{X} 3 \mathrm{D}$ scene files of the fitted result

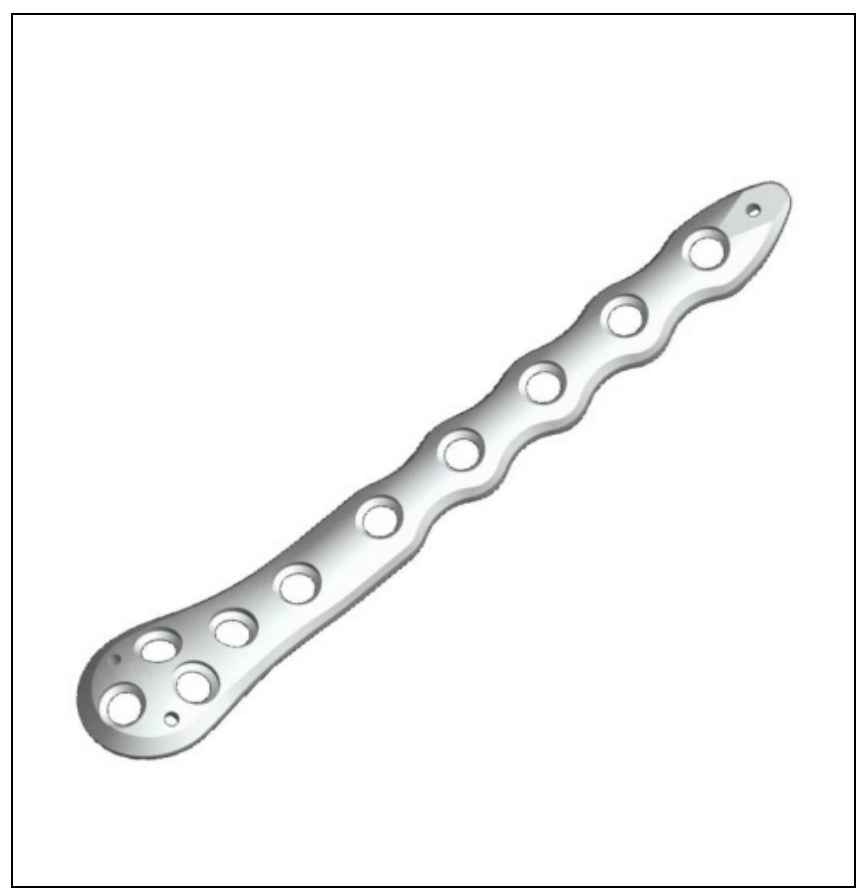

Fig. (8). Configuration of the designed implant in a computer sketch (five-hole version).

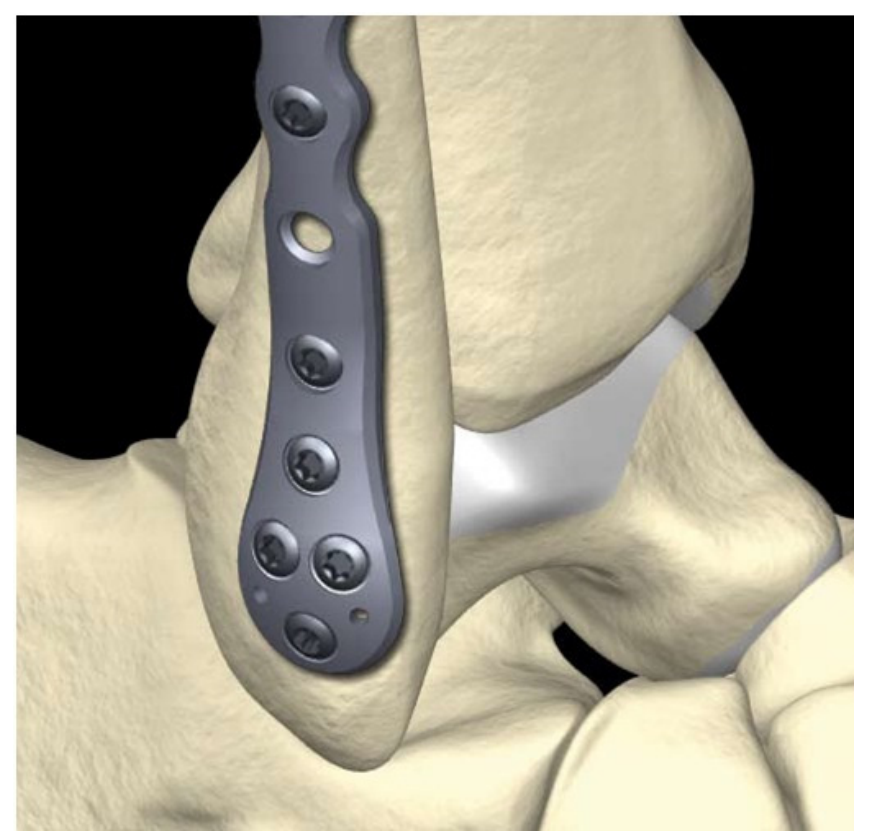

Fig. (9). Virtual testing with the help of the VDBD showed that the plate is usable for both left and right (pictured above the right ankle).

There was no statistical difference found comparing the fitting distance within the two populations. First order statistics were evaluated for the Caucasian and Asian group (Tables 2 and 3). In Fig. (10), the minimum mean and maximum fitting errors are displayed graphically. 
Table 2. First Order Statistics Obtained for the Caucasian Population

\begin{tabular}{|c|c|c|c|c|}
\hline$[\mathbf{m m}]$ & Min & Max & Mean & StdDev \\
\hline \hline Min stats & $4.7 \mathrm{e}-04$ & 1.59 & 0.54 & 0.38 \\
\hline Max stats & 0.68 & 4.37 & 3.13 & 0.64 \\
\hline Mean stats & 0.16 & 2.74 & 1.61 & 0.54 \\
\hline
\end{tabular}

Every column corresponds to min, max, and mean values across implants. Rows correspond to min, max, mean and standard deviation within the obtained min, max, or mean implant fitting.

Table 3. First Order Statistics Obtained for the Asian Population

\begin{tabular}{|c|c|c|c|c|}
\hline$[\mathbf{m m}]$ & Min & Max & Mean & StdDev \\
\hline \hline Min stats & $1.52 \mathrm{e}-05$ & 1.31 & 0.33 & 0.29 \\
\hline Max stats & 1.27 & 4.36 & 3.04 & 0.64 \\
\hline Mean stats & 0.31 & 2.40 & 1.44 & 0.41 \\
\hline
\end{tabular}

Every column corresponds to min, max, and mean values across implants. Rows correspond to min, max, mean and standard deviation within the obtained min, max, or mean implant fitting.

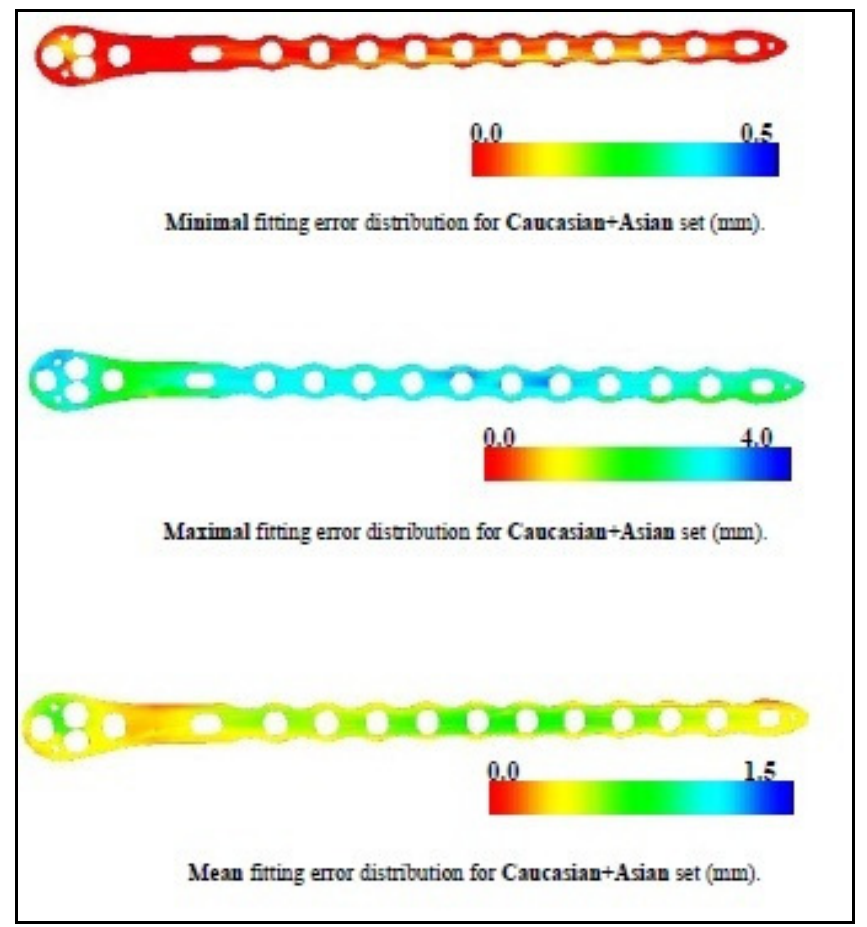

Fig. (10). The determined fitting errors as means of both subpopulations.

\section{Biomechanical Evaluation}

The fatigue strength of the VariAx Distal Lateral Fibula Platewas significantly higher than the fatigue strength of the LCP 1/3 Tubular Plate (Fig. 11).

The cut-out strengthfor VariAx Distal Lateral Fibula Plate constructs washigherthan for LCP 1/3 Tubular Plate constructs (Fig. 12). All VariAx constructs sustained a higher number of load cycles than the LCP constructs.

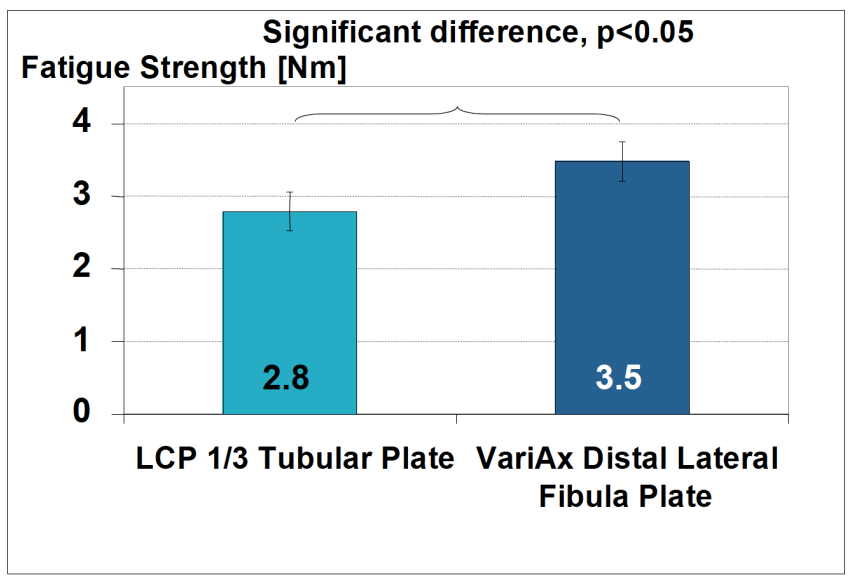

Fig. (11). Statistical evaluation of the fatigue testing showing significantly higher fatigue strength for the anatomically shaped plate.

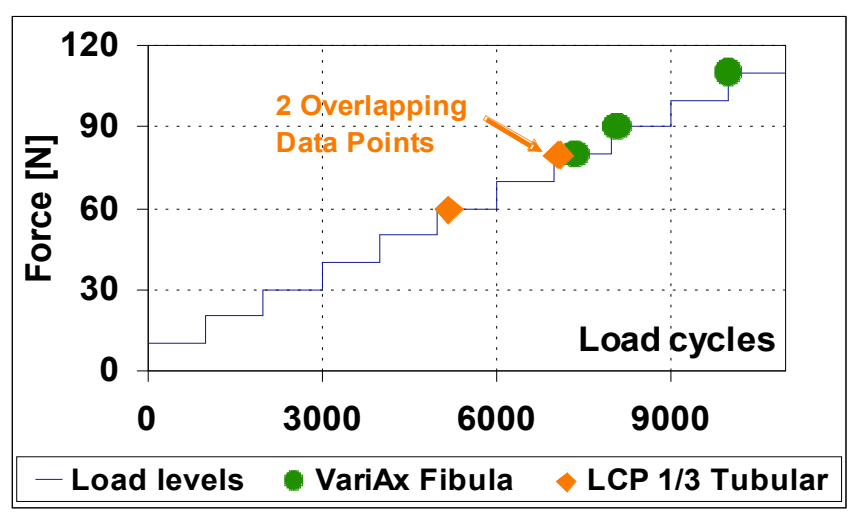

Fig. (12). Per load stage 1'000 load cycles were applied before increasing the force. Results of the anatomical VariAx plate (green) and the LCP (orange) are displayed.

\section{Clinical Implant Application}

In cases of bimalleolar fractures $(49.1 \%)$ cannulated screws (10 cases) or additional plates (1 case) have been used for the medial malleolus.

In $42.1 \%$ of all cases, the syndesmosis was found to be ruptured and the involvement of tibia fracture has been reported in $47.4 \%$ of all cases. In 21 cases a syndesmosis screw has been placed inside the plate.

The main focus of the clinical part of this study was to evaluate in how many cases and how severe the anatomically pre-contouredVariAx plate had to be bent to fit the patient's anatomy well. In 47 cases $(82.5 \%)$ there was no further bending necessary. Only in two cases "a lot of bending" was found to be necessary by the performing surgeons (Fig. 13). A later case evaluation showed that these surgeons attempted to mount the lateral fibular plate in a dorsolateral position.

As there is no direct measurement of the soft tissue coverage for an orthopaedic implant, surgeons had to estimate the adequacy of the achieved coverage. Overall 87.7 $\%$ were very satisfied or satisfied (see Fig. 14).

Finally the surgeons' satisfaction with the new type of implant has been investigated. Overall $87 \%$ of the surgeons were "very satisfied" or "satisfied" with the VariAx Fibula Plate (Fig. 15). 


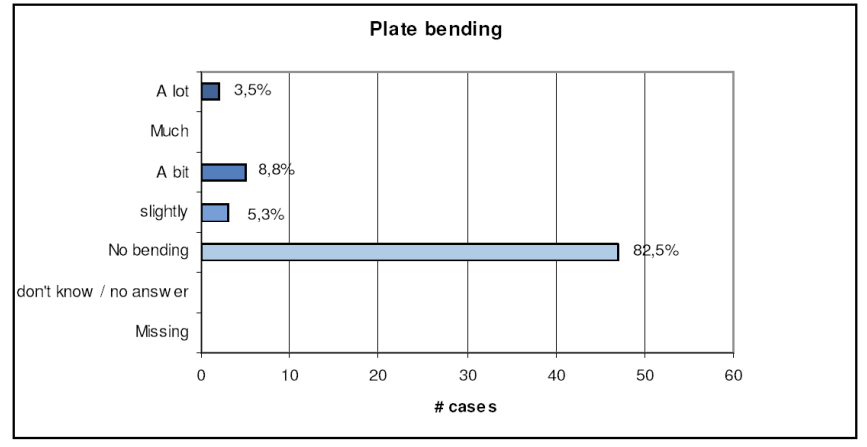

Fig. (13). Surgeons self-estimation values of plate bending requirements during the surgery.

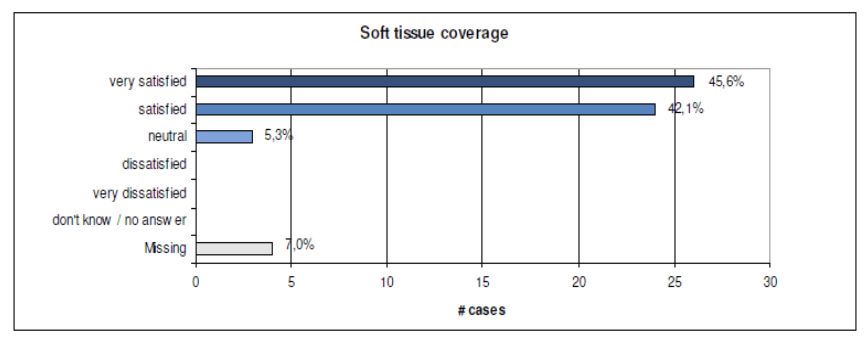

Fig. (14). Surgeons' estimations of soft tissue coverage with the new VariAx Fibula Plate.

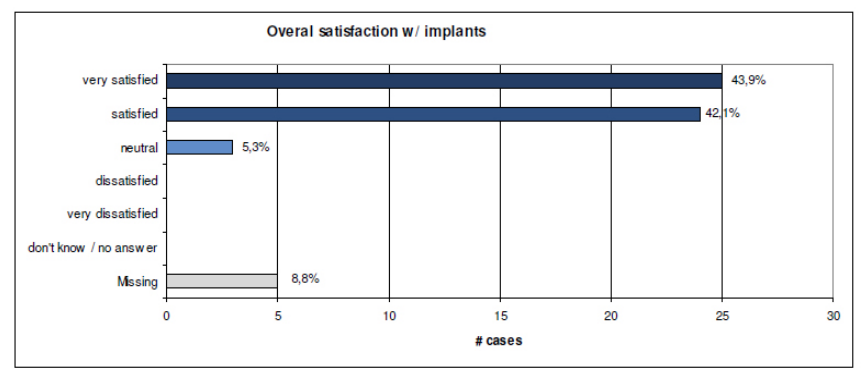

Fig. (15). After the procedure every surgeon had to state his satisfaction with the novel implant ranging from "very satisfied" to "very dissatisfied" on a pseudonymised score sheet.

\section{DISCUSSION}

In the past it has been found that "anatomically designed implants" have in fact a high degree of mismatch with the actual human anatomy in the area they were supposed to fit $[2,14]$. In our study not only was it possible to design a novel implant shape based on anatomical evidence and computational means. It was also possible to validate the implant fit for different ethnical subgroups. To our knowledge there has been no other study published investigating this topic.

In this project a technology has been developed and applied that can lead to better implant design in terms of both morphology and biomechanical performance. This has been achieved by building digital models of human anatomy and biomechanical properties, which allow virtual implant testing to be performed across a target population.

In the future virtual testing of new implants might replace cadaver testing. Furthermore, being able to study the whole range of bone shapes and densities of the target population can lead to better fitting implants as well as a considerable cost reduction during the design process [20].
The next step in this study was tocompare the biomechanical properties of the plate-screw construct with an implant that is in commonly used in the area of distal fibular fractures. The biomechanical properties of the newly designed VariAx Fibula Plate were found to be superior. The fatigue strength is influenced by the implant material and design. In addition, a proper bone fit was found to have a positive influence on the construct strength. It enhances the load distribution and decreases stress peaks [21].

It has been proven that a virtual design process can significantly increase the biomechanical stability of such implants whilst the implant volume itself can be decreased drastically [22]. The main factors for higher cut-out strength are the number of screws and the screw orientation in the distal fragment. Again the anatomical fit of the VariAx plate potentially leads to an increased cut-out strength, as the bone segment is well-supported by the plate. The clinical significance of these results is that the VariAx Distal Lateral Fibula Plate shows a higher cut-out strength than the commonly used LCP 1/3 Tubular Plate. As the newly developed plate also has other distinct differences to the second implant tested (multidirectional locking screws and two parallel screws in the distal area) no clear statement can be given if the superior stability stems from the anatomical shape or the other features described above.

In a prospective multinational trial of 57 cases it could be proven that the shape fit from virtual construction and validation also was transferable to the day to day clinical situation with a very good intra-operative anatomical fit requiring minimal or no bending. A major difference to previous universally applicable implants is the precontouring for a distinct anatomical site. In our clinical trial the only cases of mismatch occurred when surgeons attempted to use the implant in a different than the intended location: namely the dorsal distal fibula instead of the distal lateral. In the future implant manufacturers will need to make surgeons aware of this restriction.

Prospective clinical trials are now required to investigate if the newly developed anatomical implant will lead to a better clinical outcome.

\section{CONCLUSION}

An anatomical database is a powerful research tool for different fields of medicine. In traumatology and orthopaedics it offers the possibility to design implants on evidence based methods. In anatomy, scientific findings can be validated against a large database.

In the future biomechanical studies might be performed entirely digitally. In the field of forensic medicine, singular measurements can be compared to an extensive digital database calculating the most likely age span, gender, ethnical background or size.

The present study showed that an evidence based approach to implant design and manufacturing is possible. In the future these type of novel implants will not only be anatomically but also biomechanically optimized [1].

\section{ACKNOWLEDGEMENT}

Declared None. 


\section{CONFLICT OF INTEREST}

Declared None.

\section{REFERENCES}

[1] Nobari S, Katoozian HR, Zomorodimoghadam S. Threedimensional design optimisation of patient-specific femoral plates as a means of bone remodeling reduction. Comput Methods Biomech Biomed Engin 2010; 13(6): 819-27.

[2] Goyal KS, Skalak AS, Marcus RE, Vallier HA, Cooperman DR. Analysis of anatomic periarticular tibial plate fit on normal adults. Clin Orthop Relat Res 2007; 461: 245-57.

[3] Queitsch C, Kienast B, Voigt C, Gille J, Jürgens C, Arndt PS. Treatment of posttraumatic sternal non-union with a locked sternum-osteosynthesis plate (TiFix). Injury 2011; 42(1): 44-6.

[4] Meiners J, Faschingbauer M, Voigt C, Jürgens C, Schulz AP. Polyaxial locked implants in the treatment of type vancouver B1 periprosthetic fractures of the femur: retrospective clinical examination in 58 cases with review of the literature. Eur J Trauma Emerg Surg 2010; 36(1): 53-9.

[5] Kienast B, Gille J, Queitsch C, et al. Early weight bearing of calcaneal fractures treated by intraoperative 3D-fluoroscopy and locked-screw plate fixation. Open Orthop J 2009; 3: 69-74.

[6] Faschingbauer M, Kienast B, Schulz AP, Vukelic R, Meiners J. Treatment of distal lower leg fractures: results with fixed-angle plate osteosynthesis. Eur J Trauma Emerg Surg 2009; 35(6): 513-9.

[7] Schulz AP, Fuchs S, Simon L, Seide K, Paech A, Queitsch C. Severe fracture of the tibial pilon: Results with a multidirectional self-locking osteosynthesis plate utilizing a two-stage procedure. Eur J Trauma Emerg Surg 2008; 34(4): 391-6.

[8] Gille J, Schulz AP, Queitsch C, Paech A, Jurgens C. Initial results of the s3-humerus plate. Open Orthop J 2008; 2: 133-6.

[9] Ehlinger M, Adam P, Bonnomet F. Minimally invasive locking screw plate fixation of non-articular proximal and distal tibia fractures. Orthop Traumatol Surg Res 2010; 96(7): 800-9.

[10] Apivatthakakul T, Arpornchayanon O, Bavornratanavech S. Minimally invasive plate osteosynthesis (MIPO) of the humeral shaft fracture. Is it possible? A cadaveric study and preliminary report. Injury 2005; 36(4): 530-8.

[11] Apivatthakakul T, Chiewcharntanakit S. Minimally invasive plate osteosynthesis (MIPO) in the treatment of the femoral shaft fracture where intramedullary nailing is not indicated. Int Orthop 2009; 33(4): 1119-26.

[12] Endo H, Asaumi K, Mitani S, et al. The minimally invasive plate osteosynthesis (MIPO) technique with a locking compression plate for femoral lengthening. Acta Med Okayama 2008; 62(5): 333-9.

[13] Gupta RK, Rohilla RK, Sangwan K, Singh V, Walia S. Locking plate fixation in distal metaphyseal tibial fractures: series of 79 patients. Int Orthop 2010; 34(8): 1285-90

[14] Schmutz B, Wullschleger ME, Noser H, Barry M, Meek J, Schütz MA. Fit optimisation of a distal medial tibia plate. Comput Methods Biomech Biomed Eng 2011; 14(4): 359-64.

[15] Kozic N, González Ballester M, Tannast M, Nolte L, Reyes M. Statistical Shape Space Analysis Based on Level Sets. Medical Imaging and Augmented Reality: Springer Berlin/Heidelberg 2008, p. $160-7$.

[16] Specchiulli F, Mangialardi R. The surgical treatment of malleolar fractures: long-term results. Chir Organi Mov 2004; 89(4): 313-8.

[17] Kozic N, Weber S, Büchler P, et al. Optimisation of orthopaedic implant design using statistical shape space analysis based on level sets. Med Image Anal 2010; 14(3): 265-75

[18] Schulz AP, Faschingbauer M, Jürgens C. Sternal non-uniondevelopment of a novel fixation device. Injury Extra 2005; 36: 56972.

[19] Lindsjo U. Operative treatment of ankle fracture-dislocations. A follow-up study of 306/321 consecutive cases. Clin Orthop Relat Res 1985; 199: 28-38.

[20] Little RE. Tables for estimating median fatigue limits. IEEE Trans Reliab 1981; R-30(5): 415

[21] Bonaretti S, Reimers N, Reyes M, Joensson A, Nolte L, Büchler P. Assessment of peri-articular implant fitting based on statistical finite element modeling. 2008 miccai workshop - computational biomechanics for medicine. New York, USA: Insight 2008.

[22] Lovald ST, Wagner JD, Baack B. Biomechanical optimization of bone plates used in rigid fixation of mandibular fractures. J Oral Maxillofac Surg 2009; 67(5): 973-85. 